PROCEEDINGS OF THE

AMERICAN MATHEMATICAL SOCIETY

Volume 134, Number 6, Pages 1681-1686

S 0002-9939(05)08225-0

Article electronically published on December 2, 2005

\title{
UNIQUENESS FOR MULTIPLE TRIGONOMETRIC AND WALSH SERIES WITH CONVERGENT REARRANGED SQUARE PARTIAL SUMS
}

\author{
J. MARSHALL ASH AND SH. T. TETUNASHVILI
}

(Communicated by Andreas Seeger)

\begin{abstract}
If at each point of a set of positive Lebesgue measure every rearrangement of a multiple trigonometric series square converges to a finite value, then that series is the Fourier series of a function to which it converges uniformly. If there is at least one point at which every rearrangement of a multiple Walsh series square converges to a finite value, then that series is the Walsh-Fourier series of a function to which it converges uniformly.
\end{abstract}

\section{INTRODUCTION}

The basic uniqueness results for one-dimensional trigonometric series have been successfully generalized to rectangularly convergent multiple trigonometric series and also to spherically convergent multiple trigonometric series [AFR, B, T]. For the case of square convergent multiple trigonometric series, only uniqueness results involving extremely strong hypotheses are known. Here is a result of that sort that comes to mind.

Proposition 1. If a multiple trigonometric series is absolutely convergent at each point of a set of positive Lebesgue measure and square convergent to zero almost everywhere, then all its coefficients are zero.

The proof is not quite trivial, because the usual definition of absolute convergence involves some clustering of terms. For example,

$$
\sum_{m, n} a_{m n} \cos m x \cos n y+b_{m n} \cos m x \sin n y+c_{m n} \sin m x \cos n y+d_{m n} \sin m x \sin n y
$$

is said to converge absolutely if

$$
\sum_{m, n}\left|A_{m n}(x, y)\right|
$$

Received by the editors January 4, 2005.

2000 Mathematics Subject Classification. Primary 42B99, 42B05, 42C20; Secondary 42C10, $42 \mathrm{~A} 63$.

Key words and phrases. Uniqueness, multiple trigonometric series, multiple Walsh series, rearrangements.

This research was partially supported by NSF grant DMS 9707011 and a grant from the Faculty and Development Program of the College of Liberal Arts and Sciences, DePaul University. 
is finite, where

$$
\begin{aligned}
A_{m n}(x, y)= & a_{m n} \cos m x \cos n y+b_{m n} \cos m x \sin n y \\
& +c_{m n} \sin m x \cos n y+d_{m n} \sin m x \sin n y .
\end{aligned}
$$

To see how this is proved, look at the latter part of the proof of Theorem 2 below. A result in the same spirit as this proposition can be found in reference AT.

Professor N. Nadirashvili asked the first author whether the square convergence to zero of every rearrangement of a multiple trigonometric series at every point forces all coefficients to be zero. This article provides a positive answer. The fundamental idea is that the good behavior of all rearrangements is strongly connected to absolute convergence.

The square partial sums of a multiple series are defined by means of an increasing sequence of nested cubes such that (1) each contains finitely many lattice points and (2) their union is the entire lattice. Actually, our results are true for any summing method defined by any family of nested sets of lattice points with properties (1) and (2). Furthermore, the following strengthening of Professor Nadirashvili's conjecture is immediate from Theorem 2 below.

Corollary 1. If a multiple trigonometric series has the property that for every point of a set of positive measure and for each rearrangement the set of square partial sums of the rearranged series is bounded and if the original series square converges to 0 on a dense set, then every coefficient is 0 .

In fact, it is enough for the first hypothesis to be satisfied only for those rearrangements which only permute clusters such as $A_{m n}(x, y)$ and not individual terms such as $a_{m n} \cos m x \cos n y$.

The corresponding multiple Walsh series uniqueness result only requires the rearrangements to behave well at a single point. From Theorem 3 we easily get this.

Corollary 2. Suppose that a multiple Walsh series is square convergent to 0 almost everywhere and also that there is at least one point at which each rearrangement of that series has bounded partial sums. Then all the coefficients of the series are 0.

\section{RESUlTS}

Let $d \geq 1$ be a natural number, let $\mathbb{R}^{d}$ be Euclidean space of dimension $d$, and let $\mathbb{Z}_{0}^{d}$ be the points of $\mathbb{R}^{d}$ with nonnegative integer coordinates. Let $\Omega^{d}=\{\Omega(N)\}_{N=0}^{\infty}$ be an increasing sequence of finite subsets of $\mathbb{Z}_{0}^{d}$. Explicitly,

(1) $\Omega(0) \subset \Omega(1) \subset \cdots \subset \Omega(N) \subset \cdots$, and

(2) $\cup_{N=0}^{\infty} \Omega(N)=\mathbb{Z}_{0}^{d}$.

Consider a $d$-multiple numerical series

$$
\sum_{n \geq 0} a_{n}
$$

where $n \geq \mathbf{0}$ means $n=\left(n_{1}, \ldots, n_{d}\right), \mathbf{0}=(0, \ldots, 0)$ and $n_{i} \geq 0, i=1, \ldots, d$.

Let $S_{N}$ be the sum of those terms of the series (2.1) with indices in $\Omega(N)$ so that

$$
S_{N}=\sum_{n \in \Omega(N)} a_{n}
$$


We denote a rearrangement of the points of $\mathbb{Z}_{0}^{d}$ by $\sigma$, so that $\sigma$ is a one-to-one correspondence from $\mathbb{Z}_{0}^{d}$ to itself.

Definition 1. The series (2.1) $\Omega^{d}$ converges to the number $s$ if $\lim _{N \rightarrow \infty} S_{N}=s$.

Note that both square convergence and spherical convergence are examples of $\Omega^{d}$ convergence. We denote the trigonometric system defined on the torus $\mathbb{T}=[0,1)$ by $T^{1}=\left\{t_{i}(\tau)\right\}_{i=0}^{\infty}$, where $t_{0}(\tau)=1, t_{2 i-1}(\tau)=\sqrt{2} \cos i 2 \pi \tau, t_{2 i}(\tau)=\sqrt{2} \sin i 2 \pi \tau, i=$ $1,2, \ldots$, and we denote the multiple trigonometric system defined on the $d$-torus $\mathbb{T}^{d}$ by $\left\{t_{n}(x)\right\}_{n \geq 0}=\left\{\prod_{j=1}^{d} t_{n_{j}}\left(x_{j}\right)\right\}_{n \geq 0}$. Then a $d$-multiple trigonometric series may be written $T(x)=\sum_{n \geq 0} a_{n} t_{n}(x)$. For any $m \geq \mathbf{0}$, denote the sum of all terms with the same multifrequency $m$ (if no $m_{i}=0$, there are $2^{d}$ of these) by $A_{m}(x)$; thus

$$
A_{m}(x)=\sum_{2 m-1 \leq n \leq 2 m} a_{n} t_{n}(x),
$$

where $2 m=\left(2 m_{1}, \ldots, 2 m_{d}\right)$ and $\mathbf{1}=(1, \ldots, 1)$. When our rearrangements are specialized to the trigonometric case, it will be sufficient for our purposes to demand good behavior only for all rearrangements that move all the $A_{m}$ as blocks. For this reason we will also write the multiple trigonometric series as

$$
T(x)=\sum_{m \geq 0} A_{m}(x),
$$

its $\Omega$ partial sums as

$$
T_{N}(x)=\sum_{m \in \Omega(N)} A_{m}(x),
$$

and its rearranged partial sums as

$$
T_{N}^{\sigma}(x)=\sum_{m \in \Omega(N)} A_{\sigma(m)}(x) .
$$

The $d$-multiple Walsh system is defined on $\mathbb{T}^{d}$ by

$$
\left\{w_{n}(x)\right\}_{n \geq \mathbf{0}}=\left\{\prod_{j=1}^{d} w_{n_{j}}\left(x_{j}\right)\right\}_{n \geq \mathbf{0}},
$$

where for each integer $\ell=\sum_{i=0}^{k} \epsilon_{i} 2^{i}$, each $\epsilon_{i}=0$ or 1 and each $x=. x_{1} x_{2} \ldots$ (binary decimal expansion), $w_{\ell}(x)=(-1)^{\sum_{i=0}^{k} \epsilon_{i} x_{i}}$.

As with the trigonometric case, we have a multiple Walsh series

$$
W(x)=\sum_{n \geq 0} a_{n} w_{n}(x),
$$

its $\Omega$ partial sums

$$
W_{N}(x)=\sum_{n \in \Omega(N)} a_{n} w_{n}(x),
$$

a rearrangement of this Walsh series

$$
W^{\sigma}(x)=\sum_{n \geq \mathbf{0}} a_{\sigma(n)} w_{\sigma(n)}(x),
$$


and the partial sums of that rearranged series

$$
W_{N}^{\sigma}(x)=\sum_{n \in \Omega(N)} a_{\sigma(n)} w_{\sigma(n)}(x) .
$$

Theorem 1. If the numerical series $S=\sum_{n \in Z_{0}^{d}} a_{n}$ has the property that for any permutation $\sigma$

$$
\sup _{N}\left|S_{N}^{\sigma}\right|=\sup _{N}\left|\sum_{n \in \Omega(N)} a_{\sigma(n)}\right|=B(\sigma)<\infty
$$

then

$$
\sum_{n \geq 0}\left|a_{n}\right|<\infty
$$

Remark 1. Condition (2.10) is a weakening of the condition that all rearrangements of $\sum a_{n}$ are $\Omega^{d}$ convergent to finite values. The latter condition is itself a weakening of the condition that all rearrangements of $\sum a_{n}$ are $\Omega^{d}$ convergent to a single finite value. Please keep this remark in mind when looking at the two corollaries above and the two theorems below.

Proof. Let $P$ be the set of indices corresponding to the nonnegative coefficients of $T(x), P=\left\{n \in Z_{0}^{d}: a_{n} \geq \mathbf{0}\right\}$, and let $Q=\left\{n \in Z_{0}^{d}: a_{n}<\mathbf{0}\right\}$ so that $Z_{0}^{d}$ is the disjoint union of $P$ and $Q$. If $\sum_{n \in P} a_{n}<\infty$ and $\sum_{n \in Q} a_{n}>-\infty$, then the conclusion (2.11) is clear. If the conclusion is false, replacing $S$ by $-S$ if necessary, we may assume that

$$
\sum_{n \in P} a_{n}=\infty
$$

We will construct a rearrangement $\sigma$ that contradicts the hypothesis (2.10). Enumerate the terms of $S$ with indices in $P$ so that the nonnegative terms of $S$ are $\left\{a_{n(0)}, a_{n(1)}, \ldots\right\}$ and write the strictly negative terms of $S$ as $\left\{a_{m(0)}, a_{m(1)}, \ldots\right\}$. Also order the points of $Z_{0}^{d}\{k(0), k(1), \ldots\}$ by placing first the points in $\Omega(0)$ (in any order), next the points of $\Omega(1) \backslash \Omega(0)$ (in any order), next the points of $\Omega(2) \backslash \Omega(1)$ (in any order), and so on. We define $\sigma$ as follows. Take the first negative term: $a_{\sigma(k(0))}=a_{m(0)}$. Now take nonnegative terms until the sum is at least 1 and we are at the end of an $\Omega$ block: set $a_{\sigma(k(1))}=a_{n(0)}, a_{\sigma(k(2))}=a_{n(1)}, \ldots, a_{\sigma\left(k\left(k_{1}\right)\right)}=$ $a_{n\left(k_{1}-1\right)}$. Here $k_{1}$ has been picked so that $S_{N_{1}}^{\sigma} \geq 1$, where $\Omega\left(N_{1}\right)$ contains exactly $k_{1}$ lattice points. Next take a second negative term: $a_{\sigma\left(k\left(k_{1}+1\right)\right)}=a_{m(1)}$ and then pick enough nonnegative terms until the sum is at least 2 , and we are at the end of another $\Omega$ block: $a_{\sigma\left(k\left(k_{1}+2\right)\right)}=a_{n\left(k_{1}+1\right)}, \ldots, a_{\sigma\left(k\left(k_{2}\right)\right)}=a_{n\left(k_{2}-2\right)}$. Here $k_{2}$ has been picked so that $S_{N_{2}}^{\sigma} \geq 2$, where $\Omega\left(N_{2}\right)$ contains exactly $k_{2}$ lattice points. Proceed inductively, always picking first one more negative term (if the list of negative terms is finite and the negative terms have already been exhausted, just skip this step) and then picking enough nonnegative terms to guarantee that $S_{N_{k}}^{\sigma} \geq k$ for every $k$. Then

$$
\sup _{N}\left|S_{N}^{\sigma}\right| \geq \sup _{k}\left|S_{N_{k}}^{\sigma}\right| \geq \sup _{k} k=\infty,
$$

contradicting the hypothesis (2.10). 
Theorem 2. Let $E \subset \mathbb{T}^{d},|E|>0$ and suppose that for each $x \in E$ and each rearrangement $\sigma$ of the $A_{n}(x)$,

$$
\sup _{N}\left|T_{N}^{\sigma}(x)\right|=B(x, \sigma)<\infty .
$$

Then the series (2.3) converges $\Omega^{d}$ uniformly to a continuous function $f(x)$, and (2.3) is the Fourier series of $f(x)$.

Proof. By Theorem 1, for each $x \in E$,

$$
\sum_{m \geq 0}\left|A_{m}(x)\right|<\infty
$$

By the Denjoy-Luzin Theorem for multiple trigonometric series, it follows from this that the sum of the absolute values of all of the coefficients of the series $T(x)$ is finite $\left[\mathrm{Z}\right.$. Since each $t_{n}(x)$ is a continuous function bounded by $\sqrt{2}$, the Weierstrass $M$-test implies that $T(x)$ converges uniformly to a continuous function $f(x)$. Furthermore, uniform convergence justifies the following interchange of sum and integral:

$$
a_{m}=\sum_{n \geq \mathbf{0}} a_{m} a_{n} \int_{T^{d}} t_{m}(x) t_{n}(x) d x=\int_{T^{d}} t_{m}(x)\left(\sum_{n \geq \mathbf{0}} a_{n} t_{n}(x)\right) d x,
$$

so that $T(x)$ is the Fourier series of $f(x)$.

Theorem 3. Let there be a point $x_{\mathbf{0}} \in \mathbb{T}^{d}$ so that for every permutation $\sigma$,

$$
\sup _{N}\left|W_{N}^{\sigma}\left(x_{0}\right)\right|=B(\sigma)<\infty .
$$

Then the series (2.6) converges $\Omega^{d}$ uniformly to an integrable function $g(x)$, and (2.6) is the Fourier series of $g(x)$.

Proof. By Theorem 1,

$$
\sum_{n \geq 0}\left|a_{n} w_{n}\left(x_{0}\right)\right|<\infty
$$

But for all $n,\left|w_{n}(x)\right|=1$, so we immediately have

$$
\sum_{n \geq 0}\left|a_{n}\right|<\infty .
$$

This means that the series $W(x)$ converges uniformly to a bounded function $g(x)$. A calculation like (2.13) above shows that $W(x)$ is the Walsh-Fourier series of $g(x)$.

Remark 2. The finiteness of the sets comprising the family $\Omega^{d}$ is not essential; it is enough to assume that they are linearly ordered and increasing to all of $\mathbb{Z}^{d}$. The proof of this involves only a modification of the proof of Theorem 1 . In that proof, partition the index set $P$ into two infinite subsets $Q$ and $R$. At least one of $\sum_{n \in Q} a_{n}$ and $\sum_{n \in R} a_{n}$ must be infinite, so suppose $\sum_{n \in R} a_{n}=\infty$. Now let $\Omega(\nu)$ be the first infinite set of the family $\Omega^{d}$. Fill $\Omega(\nu)$ with the elements of $R$. Complete $\sigma$ by matching $\mathbb{Z}_{0}^{d} \backslash \Omega(\nu)$ with $N \cup Q$. The desired contradiction follows, since $\sum_{n \in \Omega(\nu)} a_{\sigma(n)}=\infty$. 
A consequence of this is that, for example, two-dimensional one-way iterated convergence can be considered an $\Omega^{2}$ method by setting

$$
\Omega((m, n))=\left\{(i, j) \in \mathbb{Z}_{0}^{2}:(i, j) \leq(m, n)\right\},
$$

where the ordering is, for example, lexicographic so that $(i, j) \leq(m, n)$ means that $i \leq m$ and if $i=m$, then $j \leq n$. Then we have $\Omega((0,0)) \subset \Omega((0,1)) \subset \Omega((0,2)) \subset$ $\cdots \subset \Omega((1,0)) \subset \Omega((1,1)) \subset \cdots$. Here

$$
\Omega(\nu)=\Omega((1,0))=\{(0, n): n=0,1,2, \ldots\} \cup\{(1,0)\}
$$

is the first infinite set of the family $\Omega^{d}$.

\section{REFERENCES}

[AFR] J. M. Ash, C. Freiling, and D. Rinne, Uniqueness of rectangularly convergent trigonometric series, Ann. of Math. 137 (1993), 145-166. MR1200079 (93m:42002)

[AT] J. M. Ash and Sh. T. Tetunashvili, New uniqueness theorems for trigonometric series, Proc. of the Amer. Math. Soc. 128 (2000), 2627-2636. MR.1657746 (2000m:42006)

[B] J. Bourgain, Spherical summation and uniqueness of multiple trigonometric series, IMRN, no. 3 (1996), 93-107. MR1383751 (97b:42022)

[T] S. Tetunashvili, On some multiple function series and the solution of the uniqueness problem for Pringsheim convergence of multiple trigonometric series, Mat. Sb. 182 (1991), 1158-1176 (Russian), Math. USSR Sbornik 73 (1992), 517-534 (English). MR1128694 (93b:42022)

[Z] Yu. A. Zaĭtsev, Cantor-Lebesgue and Fatou-Denjoy-Luzin theorems for multiple trigonometric series, Vestnik Moskov. Univ. Ser. I Mat. Mekh. 40 (1985), no. 3, 3-8, 101 (Russian), Moscow Univ. Math. Bull. 40 (1985), no. 3, 1-7 (English). MR0802606 (87a:42032)

Department of Mathematical Sciences, DePaul University, Chicago, Illinois 606143504

E-mail address: mash@math.depaul.edu

URL: http://condor.depaul.edu/ mash/

Department of Mathematics, Georgian Technical University, Kostava str. 77, 0175 Tbilisi, Republic of Georgia

E-mail address: stetun@hotmail.com 